ARTICLE

\title{
Surface-controlled reversal of the selectivity of halogen bonds
}

\author{
Jalmar Tschakert ${ }^{1,2}$, Qigang Zhong (1) 1,2, Daniel Martin-Jimenez (1) 1,2, Jaime Carracedo-Cosme (i) 3,4, \\ Carlos Romero-Muñiz (iD 4,8, Pascal Henkel2,5, Tobias Schlöder (1D) 2,5,9, Sebastian Ahles 2,6, \\ Doreen Mollenhauer ${ }^{2,5}$, Hermann A. Wegner ${ }^{2,6}$, Pablo Pou (id ${ }^{4,7}$, Rubén Pérez (1) 4,7, André Schirmeisen ${ }^{1,2}$ \& \\ Daniel Ebeling (1,2凶
}

Intermolecular halogen bonds are ideally suited for designing new molecular assemblies because of their strong directionality and the possibility of tuning the interactions by using different types of halogens or molecular moieties. Due to these unique properties of the halogen bonds, numerous areas of application have recently been identified and are still emerging. Here, we present an approach for controlling the 2D self-assembly process of organic molecules by adsorption to reactive vs. inert metal surfaces. Therewith, the order of halogen bond strengths that is known from gas phase or liquids can be reversed. Our approach relies on adjusting the molecular charge distribution, i.e., the $\sigma$-hole, by moleculesubstrate interactions. The polarizability of the halogen and the reactiveness of the metal substrate are serving as control parameters. Our results establish the surface as a control knob for tuning molecular assemblies by reversing the selectivity of bonding sites, which is interesting for future applications.

\footnotetext{
${ }^{1}$ Institute of Applied Physics (IAP), Justus Liebig University Giessen, Heinrich-Buff-Ring 16, 35392 Giessen, Germany. ${ }^{2}$ Center for Materials Research (LaMa), Justus Liebig University Giessen, Heinrich-Buff-Ring 16, 35392 Giessen, Germany. ${ }^{3}$ Quasar Science Resources S.L., Camino de las Ceudas 2, E28232 Las Rozas de Madrid, Spain. ${ }^{4}$ Departamento de Física Teórica de la Materia Condensada, Universidad Autónoma de Madrid, E-28049 Madrid, Spain. ${ }^{5}$ Institute of Physical Chemistry, Justus Liebig University Giessen, Heinrich-Buff-Ring 17, 35392 Giessen, Germany. ${ }^{6}$ Institute of Organic Chemistry, Justus Liebig University Giessen, Heinrich-Buff-Ring 17, 35392 Giessen, Germany. ${ }^{7}$ Condensed Matter Physics Center (IFIMAC), Universidad Autónoma de Madrid, E-28049 Madrid, Spain. ${ }^{8}$ Present address: Department of Physical, Chemical and Natural Systems, Universidad Pablo de Olavide, Ctra. Utrera Km. 1, E-41013 Seville, Spain. ${ }^{9}$ Present address: Institute of Nanotechnology, Karlsruhe Institute of Technology (KIT), Hermann-von-Helmholtz-Platz 1, 76344 EggensteinLeopoldshafen, Germany. ${ }^{凶}$ email: Daniel.Ebeling@ap.physik.uni-giessen.de
} 
ntermolecular halogen bonds are ideally suited for controlling molecular self-assembly and designing new materials due to their strong directionality and tunability. With this unique combination of properties halogen bonds enable molecular assemblies with manifold conformations. This is particularly interesting for broad applications in the fields of supramolecular chemistry $^{1-4}$, crystal engineering ${ }^{5-7}$, catalysis $^{8}$, and drug $\operatorname{design}^{9,10}$. This potential was gradually realized during the last two decades when the nature of the halogen bond was better understood.

Today, we know that the charge distribution of a halogen (X) that is covalently bound to an organic molecule $(\mathrm{R})$ is anisotropic. Typically, a "belt" of high electron density is observed around the $\mathrm{X}-\mathrm{R}$ bond axis, while the electron density at the cap of the halogen is significantly lower (see Fig. 1a). This region, the socalled " $\sigma$-hole", can be even positive depending on the type of halogen and the organic rest ${ }^{11}$.

The $\sigma$-hole is accountable for both the directionality of the halogen bond and its tunability. As sketched in Fig. 1b for a $\mathrm{C}_{6} \mathrm{H}_{5}-\mathrm{X} \cdots \mathrm{X}-\mathrm{C}_{6} \mathrm{H}_{5}$ contact (we indicate the non-covalent intermolecular halogen bond in the text with three dots and in the figures with dotted lines) the anisotropic charge distribution at the halogen favors an effective bonding angle of $90^{\circ}$ (see upper part), however, slightly different angles of, e.g., $120^{\circ}$ are also possible (see lower part). Both cases are typically classified in the literature as type II halogen bonds ${ }^{12}$ and in agreement with the actual IUPAC definition of the halogen bond ${ }^{13}$. So-called "head-
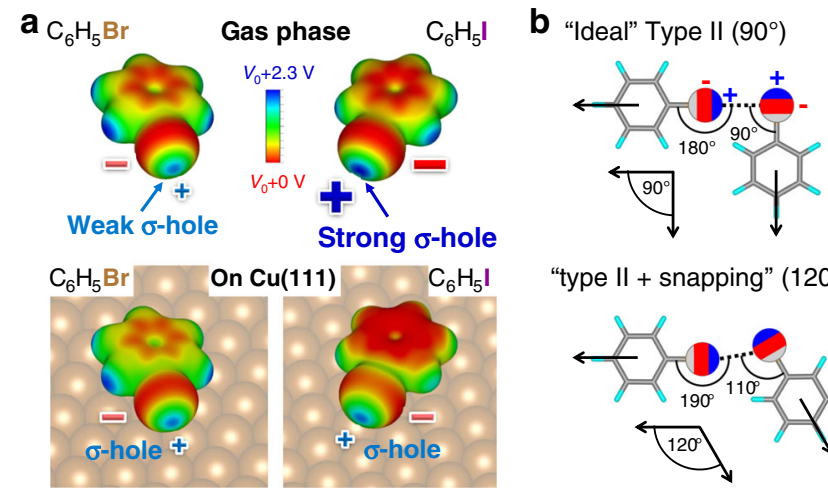

"type II + snapping" $\left(120^{\circ}\right)$
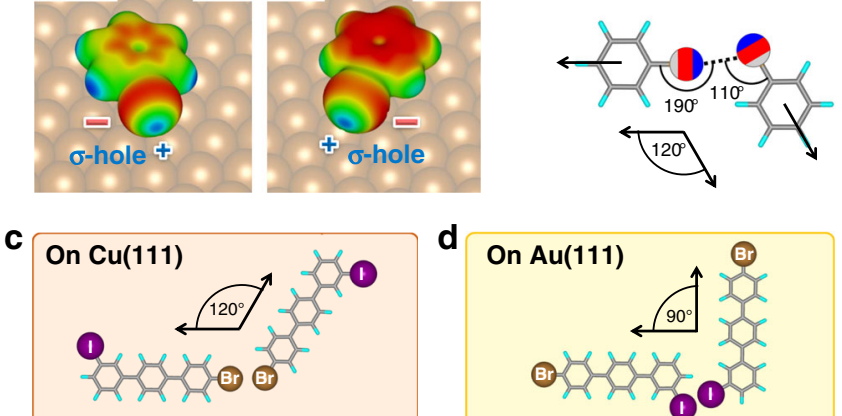

$\mathrm{Br}$...Br halogen bonds preferred

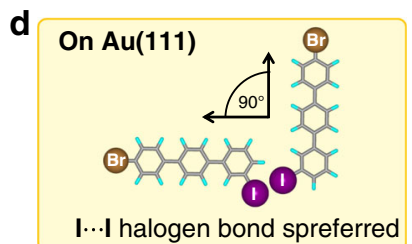

Fig. 1 Charge distribution of halogen benzenes, scheme of type II halogen bonds, and bonding configurations on $\mathrm{Cu}(111)$ and $\mathrm{Au}(111)$. a Electrostatic potential (ESP) at the $10^{-3}$ e bohr $^{-3}$ charge density isosurface as calculated by DFT of bromobenzene $\left(\mathrm{C}_{6} \mathrm{H}_{5} \mathrm{Br}\right)$ and iodobenzene $\left(\mathrm{C}_{6} \mathrm{H}_{5} \mathrm{I}\right)$ in gas phase and on $\mathrm{Cu}(111)$. The contribution of the substrate has been removed (see "Methods"). The "negative belts" and the " $\sigma$-holes" appear as red and blue regions, respectively (indicated by red "-" and blue "+" signs). b Schematic drawings of type II halogen bonds with effective bonding angles of $90^{\circ}$ (upper part) and $120^{\circ}$ (lower part), respectively. The effective bonding angles $\left(\theta_{\text {eff }}=-180^{\circ}+\theta_{1}+\theta_{2}\right)$ are measured between the $C-X$ axes as indicated by black arrows. Black dotted lines between the positive $\sigma$ hole (blue region) and the negative belt (red region) of neighboring halogens are indicating the halogen bonds. c, d Sketch of observed molecular assemblies on the reactive $\mathrm{Cu}(111)$ and the inert $\mathrm{Au}(111)$ surface indicating the control over the bonding configuration and the selectivity. on" configurations with an effective bonding angle of, or close to, $180^{\circ}$ (type I halogen bonds) are only observed in densely packed crystals since these contacts do not arise from attractive electrostatic interactions.

Intermolecular halogen bonds can be tuned by either changing the type of halogen or the rest of the molecule. The strength of the $\sigma$-hole increases with the polarizability and decreases with the electronegativity of the halogen. Hence, in gas phase it follows the order $\mathrm{I}>\mathrm{Br}>\mathrm{Cl}>\mathrm{F}^{14,15}$. This behavior is exemplarily shown in Fig. 1a for bromobenzene $\left(\mathrm{C}_{6} \mathrm{H}_{5} \mathrm{Br}\right)$ and iodobenzene $\left(\mathrm{C}_{6} \mathrm{H}_{5} \mathrm{I}\right)$ (see upper part). The $\sigma$-hole can also be effectively tuned by changing the molecular substituent that is bound to the halogen. In general, a substituent with high electronegativity will strengthen the $\sigma$-hole ${ }^{15,16}$. Therefore, e.g., the hybridization of the carbon atom that binds to the halogen plays a significant role.

Effects of different types of halogens have recently been revealed for molecular assemblies on surfaces. Using low temperature scanning probe microscopy with CO-functionalized tips ${ }^{17,18}$ it was demonstrated that even fluorinated compounds, which have an anisotropic charge distribution but do not develop a positive $\sigma$-hole due to the low polarizability of fluorine, form molecular clusters that are very similar to corresponding brominated compounds ${ }^{19-21}$. Interestingly, for brominated compounds, halogen bonded 2D molecular islands or networks with threefold symmetry have been observed on different surface materials, including $\mathrm{Cu}(111), \mathrm{Ag}(111)$, and $\mathrm{Au}(111)^{22-25}$. Halogen-bonded assemblies with tetragonal packing, however, have only been reported for relative inert substrates such as Ag (111) or $\mathrm{Au}(111)^{26-28}$, which suggests that the reactivity of the surface material influences the halogen bonds.

Understanding the influence of the surface on the halogen bonds can provide alternative strategies for tuning halogen bonds and will be useful for future applications in different fields. In the field of 2D crystal engineering such knowledge can be applied for designing molecular networks with desired structures and properties ${ }^{7}$. The catalysis of organic reactions can also benefit from this knowledge, since the strength of the halogen bond is determining its catalytic activity ${ }^{8}$. Such information is also relevant to the field of on-surface chemistry. For example, it has been shown in the literature that on-surface coupling reactions can be directed using molecular selfassembly for designing novel compounds and structures ${ }^{29,30}$. Furthermore, some on-surface reactions, such as the Ullmanntype coupling involve halogenated precursors. Information about the influence of the surface on the halogen bonds can help to better understand the pathways and mechanisms of such on-surface reactions, what is important for designing new materials with unique properties ${ }^{31-33}$.

Here, we present an approach for tuning the strength and the directionality of halogen bonds by employing molecule-substrate interactions. As depicted in Fig. 1a, the charge distributions of bromobenzene and iodobenzene change significantly after adsorption to a $\mathrm{Cu}(111)$ surface. Since this effect is much stronger for the iodobenzene than for the bromobenzene, this will undoubtedly influence the relative strength of the $\sigma$-holes. Therefore, adsorption to a relative reactive surface, such as $\mathrm{Cu}$ (111) will strongly influence the molecular assemblies and can even reverse the selectivity of bonding sites. On an inert surface, such as $\mathrm{Au}(111)$, however, the gas phase order of the strength the $\sigma$-holes is practically retained. This opens the possibility of controlling the self-assembly process by changing the surface material or the type of halogen (see sketches in Fig. 1c, d). With our approach, we gain understanding about the selectivity of halogen bonds in dependence of the substrate material and its effect on the bonding geometry, which was missing until now. 

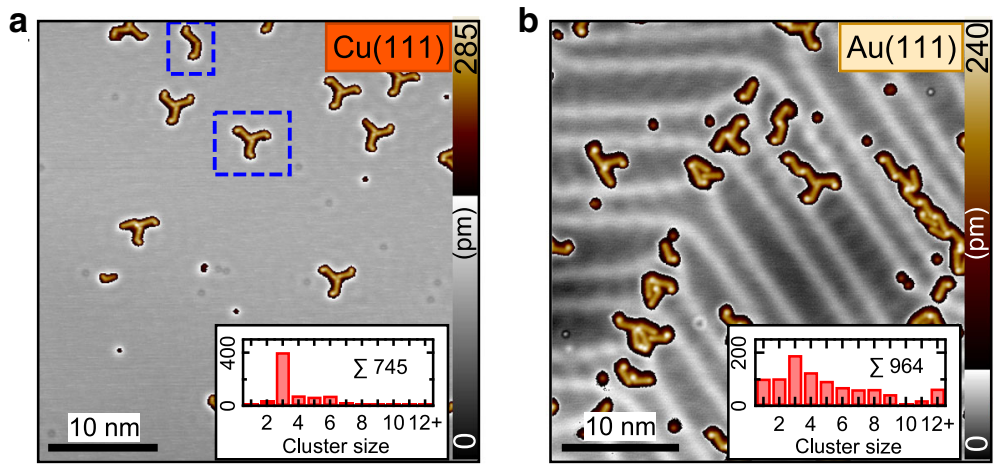

C<smiles>Brc1ccc(-c2ccc(-c3cccc(I)c3)cc2)cc1</smiles>
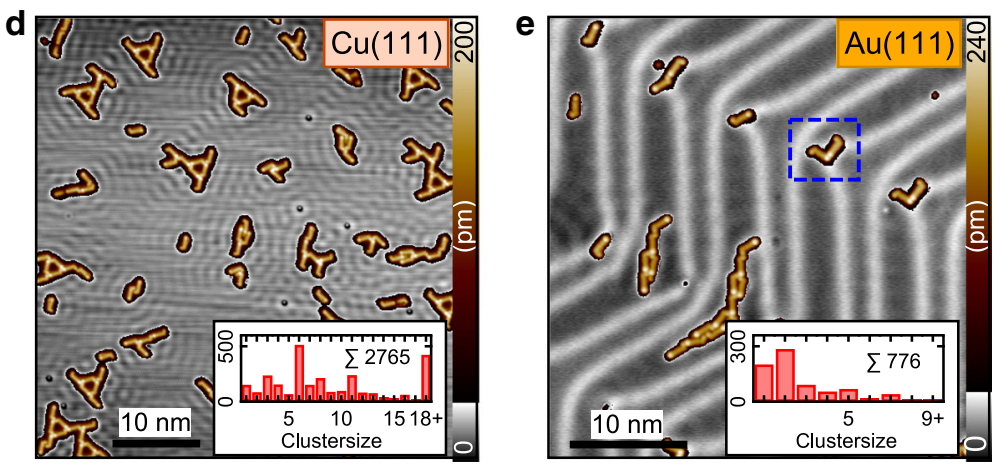

\section{f IparaBrmeta-TP}<smiles>Brc1cccc(-c2ccc(-c3ccc(I)cc3)cc2)c1</smiles>
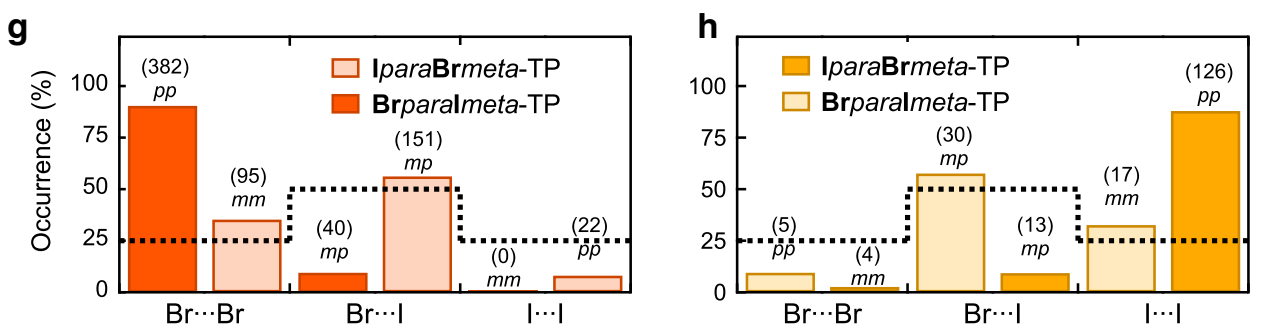

Fig. 2 Overviews of halogen bonded clusters and selectivity of halogen bonds. $\mathbf{a}, \mathbf{b}, \mathbf{d}$, e STM overview scans of Brparalmeta-TP and IparaBrmeta-TP on $\mathrm{Cu}(111)$ and $\mathrm{Au}(111)$. The insets give the total numbers of counted molecules that were found in clusters of a certain size. The dashed blue boxes indicate typical windmills and dimers as analyzed in Fig. 3. c, f Chemical structures of Brparalmeta-TP (used in $\mathbf{a}, \mathbf{b}$ ) and $\mathbf{I p a r a B r m e t a - T P}$ (used in d, e). $\mathbf{g}$, $\mathbf{h}$ Statistical distribution of $\mathrm{Br} \cdots \mathrm{Br},|\cdots|$, and $\mathrm{Br} \cdots \mid$ halogen bonds on $\mathrm{Cu}(111)$ and $\mathrm{Au}(111)$, respectively. On $\mathrm{Cu}(111)$ only dimers and trimers and on $\mathrm{Au}(111)$ only dimers are analyzed. The total number of counted halogen bonds are given in parentheses. Para-para, para-meta, or meta-meta connections are indicated by italic letters. The dashed black lines represent the statistical 25:50:25\% distribution that would be observed if $\mathrm{Br} \cdots \mathrm{Br}$, $\mathrm{Br}$...।, and I...। connections were equal in strength. Parameters: sample bias voltage $U=100 \mathrm{mV}$, tunneling current $I=10 \mathrm{pA}(\mathbf{a}, \mathbf{b}, \mathbf{e})$ and $I=100 \mathrm{pA}(\mathbf{d})$.

\section{Results and discussion}

Selectivity of halogen bonds on $\mathrm{Cu}(111)$ and on $\mathrm{Au}(111)$. All scanning tunneling microscope (STM) and AFM images presented in this work have been measured at $5.2 \mathrm{~K}$ with a lowtemperature STM/AFM (ScientaOmicron) and COfunctionalized metal tips (see "Methods"). The two structural isomers 4-bromo- $3^{\prime \prime}$-iodo-p-terphenyl (BrparaImeta-TP, see Fig. 2c) and 3-bromo-4"-iodo-p-terphenyl (IparaBrmeta-TP, Fig. 2f) have been specifically designed for this study to serve as model compounds. By attaching the bromine and iodine atoms to the para and meta position (see Fig. 2c, f) of the terphenyl backbone the two halogens are electronically decoupled by an additional phenyl ring and an unambiguous identification of molecules is possible from large area STM scans ${ }^{32}$. The latter is beneficial for obtaining reliable statistics. Furthermore, by studying both isomers effects that arise from para-para or meta-meta type connections can be evaluated. The molecules were evaporated to precooled substrates to avoid dehalogenation. Low coverages were used to obtain small cluster sizes.

Figure $2 \mathrm{a}, \mathrm{b}, \mathrm{d}$, e shows exemplary overview STM images of the two isomers BrparaImeta-TP and IparaBrmeta-TP on $\mathrm{Cu}(111)$ and $\mathrm{Au}(111)$ and statistical information about the distribution of cluster sizes (dimers, trimers, tetramers, etc.). Therefore, the total number of molecules that were found in clusters with a certain size are given in the insets. Several of these STM images were used for determining the selectivity of the halogen bonds (Fig. 2g, h). The two different substrates $\mathrm{Cu}(111)$ and $\mathrm{Au}(111)$ have been chosen as model systems for a relatively reactive and a rather inert surface, respectively. Accordingly, the $\mathrm{Cu}(111)$ surface should show a strong influence on the selectivity, while the selectivity that is known from gas phase should be retained on $\mathrm{Au}(111)$.

To ensure that influences of the surface reconstruction on $\mathrm{Au}$ (111) or the requirements for building 2D networks on $\mathrm{Cu}(111)$ are excluded, we counted only the smallest clusters. This is essential, since on $\mathrm{Cu}(111)$, clusters with four or more molecules tend to rearrange into 2D Sierpinski networks (see Fig. S1) ${ }^{25}$. On Au(111), molecular trimers are already influenced by the herringbone reconstruction. Larger clusters form braid-like structures in the $\mathrm{fcc}$ regions of the herringbone reconstruction (Fig. 2b, e).

$\mathrm{On} \mathrm{Cu}(111), \mathrm{Br} \cdots \mathrm{Br}$ connections are strongly preferred over $\mathrm{I} \cdots \mathrm{I}$ connections for both studied isomers (Fig. $2 \mathrm{~g}$ ), which is 
completely unexpected since it is well known that iodobenzene has a stronger $\sigma$-hole than bromobenzene in the gas phase (see Fig. 1a). Para-para connections are favored over meta-meta connections. In the case of BrparaImeta-TP, we count $90.5 \%$ $\mathrm{Br} \cdots \mathrm{Br}$ (para-para), $0.0 \%$ I $\cdots \mathrm{I}$ (meta-meta), and $9.5 \% \mathrm{Br} \cdots \mathrm{I}$ (para-meta) connections (see dark orange bars). For IparaBrmeta-TP, we observe $35.4 \% \mathrm{Br} \cdots \mathrm{Br}$ (meta-meta), $8.2 \%$ I $\cdots \mathrm{I}$ (para-para), and $56.3 \% \mathrm{Br} \cdots \mathrm{I}$ (meta-para) connections (see bright orange bars).

By repeating these measurements on $\mathrm{Au}(111)$, we observe a reversed selectivity of the binding sites (Fig. $2 \mathrm{~h}$ ). Here, we count $88.0 \%$ I $\cdots$ I (para-para), $2.7 \% \mathrm{Br} \cdots \mathrm{Br}$ (meta-meta), and $9.3 \%$ $\mathrm{Br}$ ‥ I (meta-para) connections in case of IparaBrmeta-TP (see dark yellow bars) and $32.7 \%$ I $\cdots \mathrm{I}$ (meta-meta), 9.6\% $\mathrm{Br} \cdots \mathrm{Br}$ (para-para), and $57.7 \% \mathrm{Br} \cdots \mathrm{I}$ (para-meta) connections in case of BrparaImeta-TP (see bright yellow bars). Due to this surface induced reversal of the selectivity, the two graphs in Fig. $2 \mathrm{~g}, \mathrm{~h}$ almost appear as mirror images of each other, which represents a remarkable control over the molecular assemblies. Please note that the reversal of bonding preference does not apply to larger clusters due to the mentioned influence of the herringbone reconstruction on $\mathrm{Au}(111)$ and the $2 \mathrm{D}$ network formation on $\mathrm{Cu}$ (111) (see Fig. S2).

Bonding configurations on $\mathrm{Cu}(111)$ and $\mathrm{Au}(111)$. The STM overview scans in Fig. 2a, d reveal that structures with effective bonding angles of $120^{\circ}$ are predominant on $\mathrm{Cu}(111)$ (see dashed blue boxes). On $\mathrm{Au}(111)$, however, effective bonding angles close to $90^{\circ}$ are observed, in particular, for molecular dimers (see dashed blue box in Fig. 2e). Next, we perform a detailed analysis of the bonding configuration and the adsorption conformations of the most abundant clusters using high resolution constantheight AFM images with CO-functionalized tips.

In Fig. 3a-f, we show a typical molecular trimer (so-called windmill) and a dimer of BrparaImeta-TP on $\mathrm{Cu}(111)$. Both structures are formed by $\mathrm{Br} \cdots \mathrm{Br}$ (para-para) connections. In Fig. 3g-i, a typical example for an I ‥I (para-para) connection of IparaBrmeta-TP on $\mathrm{Au}(111)$ is depicted. By fitting the corresponding molecular structures to the AFM images (Fig. 3b, e, h) the bonding angles and distances can be precisely determined (see zoom-ins in Fig. $3 \mathrm{c}, \mathrm{f}, \mathrm{i})^{31}$. Our findings can be summarized as follows: On $\mathrm{Cu}(111)$, bonding angles of $\theta_{1} \approx 190^{\circ}$ and $\theta_{2} \approx 110^{\circ}$ and bond lengths of $320-410 \mathrm{pm}$ are observed for typical windmills and dimers (see Figs. 3a-f and S3). The molecular connections can be classified as type II halogen bonds with an effective bonding angle of $\theta_{\text {eff }} \approx 120^{\circ}$ (cf. Fig. 1b lower part). Approximately, $95 \%$ of the molecules, align with the [112] directions (see Fig. S4), which is in agreement with previous findings for single adsorbed BrparaImeta-TP molecules on $\mathrm{Cu}$ $(111)^{32}$. This indicates a strong snapping to the threefold $\mathrm{Cu}(111)$ surface lattice due to strong molecule-substrate interactions.

On $\mathrm{Au}(111)$, the measured bonding angles for the IparaBrmeta-TP dimer are $\theta_{1} \approx 178^{\circ}$ and $\theta_{2} \approx 96^{\circ}$ (Fig. 3i). This results in an effective bonding angle of $\theta_{\text {eff }} \approx 94^{\circ}$ for the I...I (para-para) connection, which is very close to an ideal type II halogen bond (Fig. 1b). On $\mathrm{Au}(111)$, the snapping of the molecules with the $[11 \overline{2}]$ directions is much weaker than on $\mathrm{Cu}(111)$ due to the lower molecule-substrate interactions. On $\mathrm{Au}(111)$, only $70 \%$ of the single molecules and only $55 \%$ of the molecules within dimers align with the $[11 \overline{2}]$ directions (see Fig. S4). The latter number can be rationalized from Fig. $3 \mathrm{~h}$, which reveals that the left molecule aligns with the [112] direction, while the right molecule is rotated by approximately $30^{\circ}$. This indicates that the snapping to the $\mathrm{Au}(111)$ lattice is overruled by the I $\cdots \mathrm{I}$ halogen bond that favors an effective bonding angle of $90^{\circ}$.
Apparently, we also found one molecular dimer on $\mathrm{Au}(111)$ with an effective bonding angle close to $120^{\circ}$ (see Fig. S5). However, this was one of the rare $\mathrm{Br}$...Br dimers of IparaBrmeta$\mathrm{TP}$ on $\mathrm{Au}(111)$ with a meta-meta type connection $(\approx 2.7 \%$, see Fig. 2e). For this particular case, the observed angle of $120^{\circ}$ is presumably caused by the relatively weak $\mathrm{Br}$...Br halogen bond that is not able to overcome the snapping to the $\mathrm{Au}(111)$ lattice. These results highlight the strong influence of the surface material on the directionality of the halogen bonds and the bonding symmetry of the observed clusters.

Adsorption positions, conformations, and charge distributions. To identify the charge distribution of the different halogens on the surface and the formation energy of the molecular assemblies, we performed density functional theory (DFT) calculations for model systems of bromo- and iodobenzene on $\mathrm{Cu}$

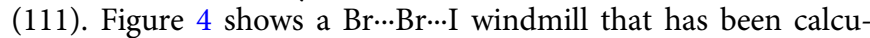
lated by DFT using $\mathrm{VASP}^{34}$ with $\mathrm{PBE}+\mathrm{D}^{35,36}$, PAW pseudopotentials 37,38 , and a plane wave cutoff of $425 \mathrm{eV}$ (see "Methods"). The adsorption positions, the conformations, and the bonding angles and distances of the three halobenzenes are in excellent agreement with our experimental findings.

More precisely, Fig. $4 \mathrm{a}$ reveals that the three halogen atoms are located close to $\mathrm{Cu}(111)$ bridge sites, the phenyl rings are located above fcc sites, and the $\mathrm{X}-\mathrm{C}$ bond axes are aligned with the crystallographic $[11 \overline{2}]$ directions (see blue arrow). This is in agreement with our experimental findings for single BrparaImeta-TP and IparaBrmeta-TP molecules on $\mathrm{Cu}(111)$ [Fig. S6 and ref. ${ }^{32}$ ] and with theoretical findings for single halobenzenes [Fig. S7 and ref. ${ }^{39}$ ]. Furthermore, the bonding angles and distances of the model system (Fig. 4b) match precisely with the values for a corresponding $\mathrm{Br} \cdots \mathrm{Br} \cdots \mathrm{I}$ windmill of IparaBrmeta-TP on $\mathrm{Cu}(111)$ (Fig. S3). In addition, the side view in Fig. $4 \mathrm{c}$ reveals that the iodine is approximately $40 \mathrm{pm}$ closer to the $\mathrm{Cu}(111)$ surface plane than the bromine.

The calculated adsorption conformation in Fig. 4c agrees with the AFM scans of single molecules [Fig. S6 and ref. ${ }^{32}$ ] and molecular clusters (Figs. 3, 4e, and S3) on $\mathrm{Cu}(111$ ), where the iodine atoms always appear significantly darker than the bromine atoms. Please note, on the inert $\mathrm{Au}(111)$ surface the iodine appears brighter than the bromine due to the almost planar adsorption conformation and the larger atomic radius of the iodine (Fig. S5) ${ }^{40}$. Since the adsorption conformation reflects the molecule-surface interactions, we also validated the image contrast by AFM simulations with a charge density based method $^{41-43}$ that some of us recently introduced (Figs. 4f, S8, and S9). The simulated AFM images are in excellent agreement with the experimental scans (Fig. 4e, f), which confirms that the iodine is closer to the $\mathrm{Cu}(111)$ surface atoms than the bromine (see details in Figs. S8 and S9). Interestingly, this detailed analysis of the image contrast also reveals that the oval appearance of the halogens in the experimental AFM scans (Figs. 3, 4e, and S6) is a direct effect of the presence of the sigma hole, which creates an anisotropic electric field sensed by the AFM probe (see Figs. S8 and S9).

The lower iodine- $\mathrm{Cu}(111)$ distance clearly indicates a relatively strong interaction between the iodine and $\mathrm{Cu}(111)$ surface atoms, while the bromine- $\mathrm{Cu}$ interaction is relatively low. As shown in Fig. $4 \mathrm{~d}$, the molecule-substrate interactions cause a charge transfer that strongly affects the charge distribution at the halogen and thereby the halogen bonds. Depicted is the differential charge density at the $10^{-4} \mathrm{ebohr}^{-3}$ isosurface, which has been calculated by subtracting the charge densities of the isolated substrate and the isolated windmill structure from the charge density of the adsorbed molecules inside the windmill on 
a

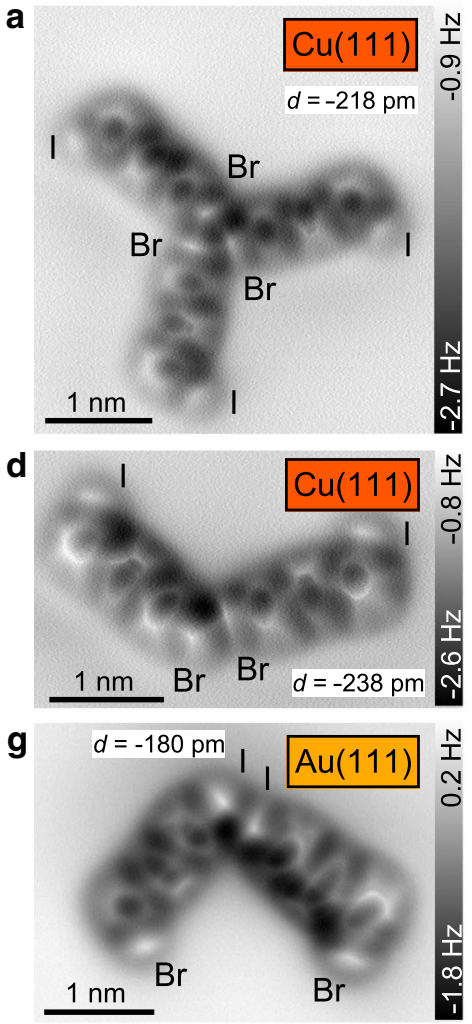

b

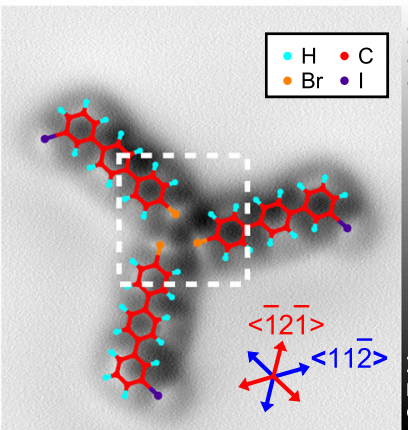

e

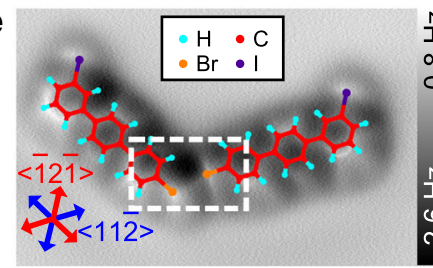

h

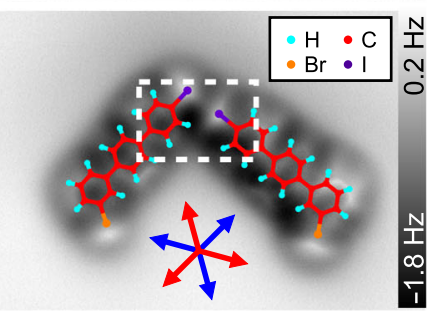

C

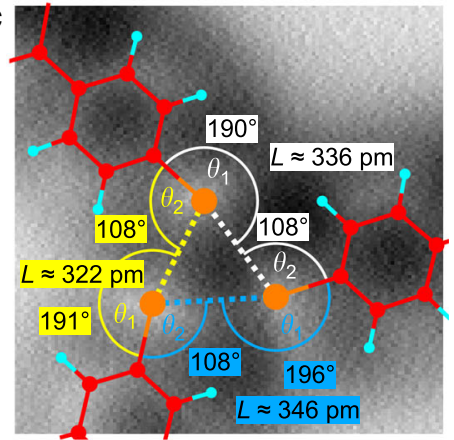

Brpara-

Imeta-TP

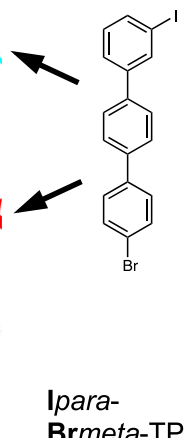

Brmeta-TP
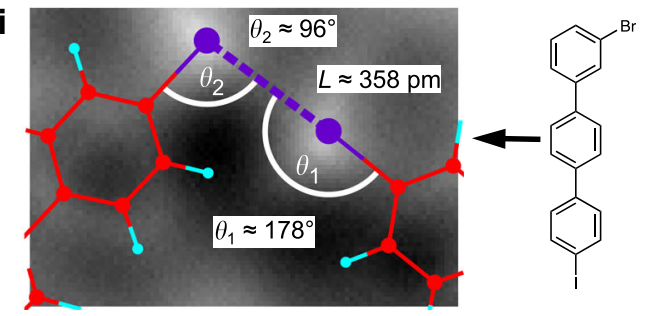

Fig. 3 Bonding configurations of typical windmills and dimers on $\mathbf{C u ( 1 1 1 )}$ and $\mathbf{A u ( 1 1 1 ) . ~ a - c ~ A F M ~ c o n s t a n t - h e i g h t ~ i m a g e s ~ o f ~ a ~} \mathrm{Br} \cdots \mathrm{Br} \cdots \mathrm{Br}$ windmill structure of

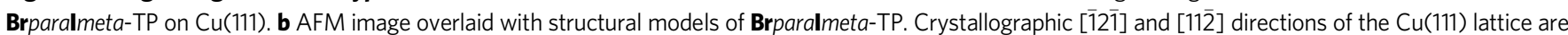
indicated with red and blue arrows, respectively. c Zoom-in to central region of the cluster with bonding angles $\theta_{1}$ and $\theta_{2}$ and bond distance $L$ of the halogen bonds. Each bromine is acting as a halogen bond donor and acceptor, respectively (see Fig. 1b). d-f AFM image and bonding configuration of a Br..Br dimer [Brparalmeta-TP on $\mathrm{Cu}(111)]$. g-i AFM image and bonding configuration of a l...I dimer [IparaBrmeta-TP on Au(111)]. Parameters: oscillation amplitude 160 pm (a, d) and $170 \mathrm{pm}(\mathbf{g})$, imaging distances are given with respect to the tunneling gap at $U=200 \mathrm{mV}(\mathbf{a}, \mathbf{d})$ and $100 \mathrm{mV}(\mathbf{g})$ and $I=10 \mathrm{pA}$.
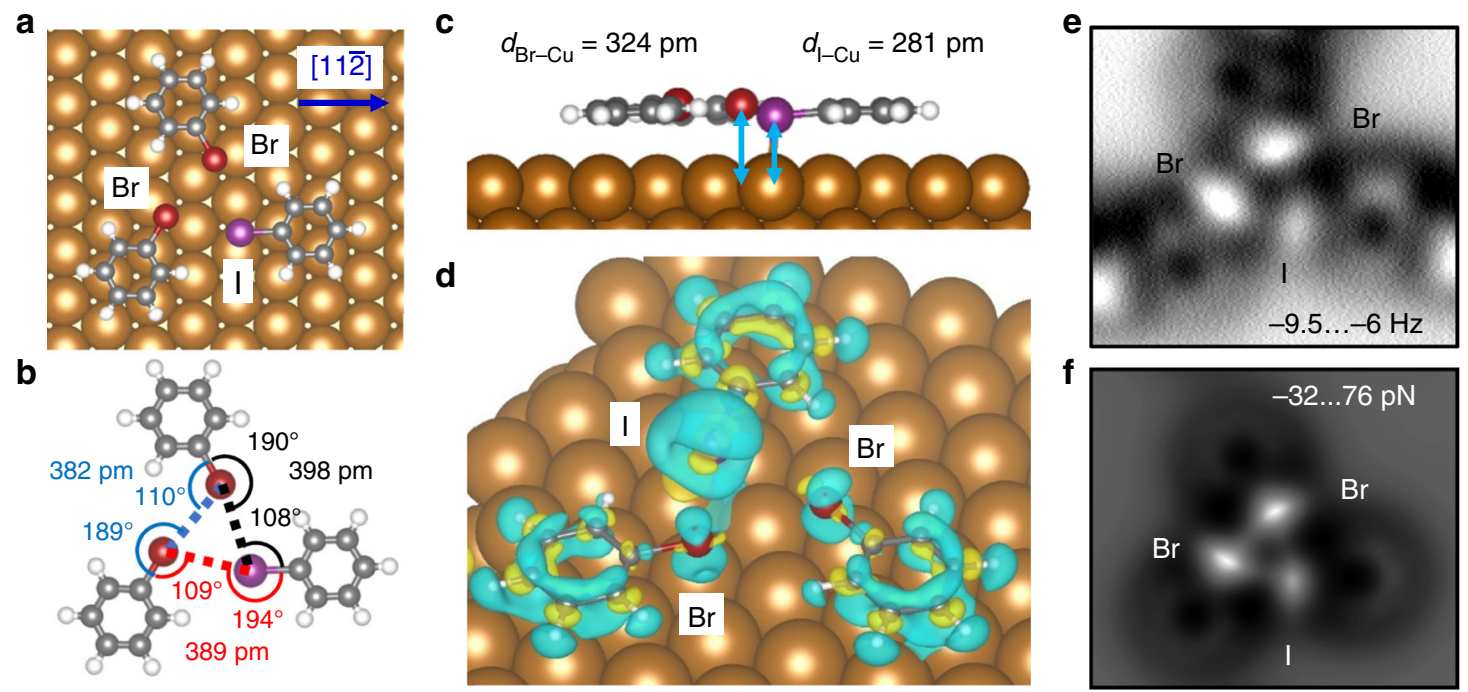

Fig. 4 DFT calculated structure and charge transfer of a halobenzene Br•••Br•••l windmill on Cu(111). a Top view of DFT calculated windmill structure consisting of two bromobenzenes and one iodobenzene on $\mathrm{Cu}(111)$ revealing the adsorption positions of the molecules. The [112] direction is indicated by the blue arrow. $\mathbf{b}$ Bonding angles and distances of structure in (a). The effective bonding angles of the three halogen bonds are $118^{\circ}, 119^{\circ}$, and $123^{\circ}$, respectively. c Side view of (a). Blue arrows indicate the distance between the halogens and the Cu(111) surface plane. $\mathbf{d}$ Differential charge density at the $10^{-4} \mathrm{e} \mathrm{bohr}^{-3}$ isosurface (yellow $=$ charge accumulation, blue $=$ charge depletion). Due to the interaction with the $\mathrm{Cu}(111)$ surface, charge is depleted from the negative belts (blue regions) and accumulated at the $\sigma$-holes (yellow regions). For iodine this effect is much stronger than for bromine. e AFM constant height image of a $\mathrm{Br} \cdots \mathrm{Br} \cdots \mathrm{I}$ windmill structure of IparaBrmeta-TP on $\mathrm{Cu}(111)$ (see details in Fig. S3). $\mathbf{f}$ Simulated force map for the structure in (a). 


\begin{tabular}{|c|c|c|}
\hline Intermolecular energies (meV) & $\mathrm{Br} \cdots \mathrm{Br} \cdots \mathrm{Br}$ & Br...Br...I \\
\hline Windmill in gas phase & -199 & -217 \\
\hline Windmill on $\mathrm{Cu}(111)$ & -100 & -70 \\
\hline
\end{tabular}

$\mathrm{Cu}(111)$. The yellow and blue regions indicate charge accumulation and depletion, respectively. The $\mathrm{Cu}(111)$ surface induces a charge depletion in the region of the negative belts (blue regions) and charge accumulation at the $\sigma$-holes (yellow regions), which results in a reduction of the strength of the halogen bonds at the surface for both halogens. Since this effect is much more pronounced for iodine than for bromine (Fig. 4d), we can rationalize the observed preference for $\mathrm{Br} \cdots \mathrm{Br}$ connections on $\mathrm{Cu}$ (111). Due to the higher polarizability of iodine, its $\sigma$-hole is weakened more strongly on the reactive $\mathrm{Cu}(111)$ surface, and consequently, its role in the cluster formation is downgraded. Interestingly, in the gas phase, the higher polarizability of iodine leads to a stronger $\sigma$-hole while on the surface the opposite is observed.

Intermolecular energies of windmill structures. Table 1 gives a comparison of the intermolecular energies of different halobenzene windmill structures in gas phase and on the $\mathrm{Cu}(111)$ surface. In gas phase the $\mathrm{Br}$... Br...I windmill is energetically more favorable than the $\mathrm{Br} \cdots \mathrm{Br} \cdots \mathrm{Br}$ structure by $18 \mathrm{meV}$. On the $\mathrm{Cu}$ (111) surface, however, the $\mathrm{Br} \cdots \mathrm{Br} \cdots \mathrm{Br}$ structure is favored by 30 $\mathrm{meV}$. The on-surface intermolecular energies were calculated by subtracting the adsorption energy of the single molecules from the total formation energy of the windmill on $\mathrm{Cu}(111)$. In the next step it is important to determine whether this relevant energy difference is only caused by the observed charge transfer or if other possible energy contributions play a distinctive role. Indeed, the energy difference could also be produced by changes in the intermolecular interaction due to both global molecular displacements and intramolecular relaxations induced by the molecule-substrate interaction (for example, the vertical shift of the I atom towards the metal substrate could cost a relatively important energy, or by the variation on the adsorption energy of each molecule as, upon windmill formation, they are not in their optimal configuration for adsorption as an isolated molecule). We have calculated these two energy contributions, obtaining values of $7 \mathrm{meV}$ for the $\mathrm{Br} \cdots \mathrm{Br} \cdots \mathrm{I}$ windmill $(0 \mathrm{meV}$ for the $\mathrm{Br} \cdots \mathrm{Br} \cdots \mathrm{Br}$ case) for the energy due to the relaxation and $6 \mathrm{meV}(3 \mathrm{meV})$ for the former contribution (see "Methods" for details). None of these contributions can explain the observed reversal of intermolecular energies on the $\mathrm{Cu}(111)$ surface, leaving the surface induced charge redistribution and its effect on the $\sigma$-hole as the main possible explanation.

Overall, the theoretical part of our study reveals a clear reduction of the formation energies of both types of windmill structures on the $\mathrm{Cu}(111)$ surface that are on the order of a hundred of $\mathrm{meV}$ (Table 1 ). This reduction is mainly caused by a charge redistribution between the $\sigma$-hole and the negative belt within the halogen atom as illustrated in Fig. $4 \mathrm{~d}$. This electronic effect is more pronounced for the iodine case, which results in lower formation energies for windmills that contain iodine. As revealed by our experimental analysis (see statistics in Fig. 2), this effect has a decisive influence on the $2 \mathrm{D}$ self-assembly process that is reflected by the different symmetries of the molecular assemblies and the reversed selectivity of the halogen bonds.
In summary, we present an approach for tuning 2D halogen bonded molecular assemblies via adsorption on relatively reactive vs. relatively inert model surfaces. An in-depth analysis of the assembly process is enabled using low temperature AFM with CO-functionalized tips and DFT calculations, which allows to precisely determine the adsorption positions and conformations of the molecules, the selectivity of the halogen bonds, the halogen bonding angles and distances, the charge distributions, and the intermolecular energies. This is essential for gaining insight into the tuning effect, since all these properties are influenced by molecule-substrate interactions. On the inert $\mathrm{Au}(111)$ surface, an almost planar adsorption conformation is observed in the AFM images, while on $\mathrm{Cu}(111)$ the iodine atoms shifts significantly toward the substrate plane due to strong halogen-substrate interactions. The observed clusters on $\mathrm{Cu}(111)$ and $\mathrm{Au}(111)$ substrates are formed via type II halogen bonds with effective bonding angles of $120^{\circ}$ and $90^{\circ}$, respectively. On the $\mathrm{Cu}(111)$ surface $\mathrm{Br} \cdots \mathrm{Br}$ halogen bonds are strongly favored, while on $\mathrm{Au}$ (111) I $\cdots$ I halogen bonds are preferred. In particular, for para-para type connections the bond selectivity is on the order of $90 \%$. The reversal of the bond selectivity is caused by halogen-substrate interactions that alter the charge distribution of the different halogens and lead to differences in the intermolecular energies. Therewith, we establish the surface material as a control knob for tuning the symmetry and the selectivity of halogen bonds, whereby the polarizability of the halogens and the reactiveness of the substrate material act as the main control parameters. These insights into the nature of halogen bonds represent an alternative approach for designing molecular assemblies. Furthermore, the conceptual idea that halogen bonds are tunable via reactive atoms in their close vicinity paves the way for future studies that address the general applicability of this phenomenon. This is appealing for applications of halogen bonds in different fields, such as crystal engineering, supramolecular chemistry, catalysis, drug design, or on-surface chemistry.

\section{Methods}

Synthesis of Brparalmeta-TP and IparaBrmeta-TP. Details about the synthesis of BrparaImeta-TP and I paraBrmeta-TP can be found in the Supplementary information. BrparaImeta-TP was synthesized according to the procedure reported in the literature ${ }^{44}$

Sample preparation. The $\mathrm{Cu}(111)$ and $\mathrm{Au}(111)$ crystals (MaTecK, Germany) were cleaned by multiple cycles of $\mathrm{Ar}+$ sputtering $\left(E=1.5 \mathrm{kV}, I=3.6 \mu \mathrm{A}, p=6 \times 10^{-6}\right.$ mbar for initial cycles and $E=0.8 \mathrm{kV}, I=1.1 \mu \mathrm{A}, p=3 \times 10^{-6}$ mbar for final cycles) and annealing ( $1000 \mathrm{~K}$ for initial cycles and $730 \mathrm{~K}$ for final cycles). The BrparaImeta-TP and IparaBrmeta-TP molecules were evaporated on the surfaces by using a home-built evaporation device held at $100-150{ }^{\circ} \mathrm{C}^{45}$. The molecules were evaporated for several seconds onto the precooled metal substrates ( $T$ below $100 \mathrm{~K}$ ) to avoid dehalogenation of the molecules.

STM/AFM. STM and AFM images were obtained under UHV conditions (pressure below $1 \times 10^{-10} \mathrm{mbar}$ ) at a temperature of $5.2 \mathrm{~K}$ with a low temperature AFM/ STM system (Scienta Omicron, Germany). In our setup the tip is grounded while the sample is connected to the bias voltage. A qPlus tuning fork sensor with a tungsten tip was used ${ }^{46}$. The tip was sharpened by voltage pulses and indentations into the respective metal surface. The AFM tip was functionalized with a $\mathrm{CO}$ molecule using standard procedures described in the literature ${ }^{47}$. Therefore, on $\mathrm{Cu}$ (111) the tip was first positioned above a CO molecule with a sample bias of $+2 \mathrm{~V}$ and a tunneling setpoint of $1 \mathrm{nA}$. Subsequently, the feedback was deactivated and the voltage was ramped from +3 to $0 \mathrm{~V}$ while the tip-substrate distance was simultaneously reduced by $100 \mathrm{pm}$. In case of $\mathrm{Au}(111)$, CO can be unintentionally picked up by scanning the surface or by voltage pulses. The tip functionalization with CO was confirmed by scanning other adsorbed CO molecules. The resonance frequencies and quality factors of the sensors were ranging from $f_{\text {res }} \approx 19$ to $27 \mathrm{kHz}$ and $Q \approx 10,000$ to 30,000 , respectively. The qPlus sensor was operated in frequency modulation mode with constant amplitude using an external phase-locked loop electronics (MFLI, Zürich Instruments, Switzerland) for obtaining both STM and AFM images. Oscillation amplitudes in the range of $40-170 \mathrm{pm}$ were used. 
First-principles calculations and AFM theoretical images. First, we characterize our halogen-molecule system by means of DFT calculations. We used the VASP package $^{34}$ with a cutoff energy of $425 \mathrm{eV}$ for the plane-wave basis set. The Perdew-Burke-Ernzerhof (PBE) functional ${ }^{35}$ supplemented by Grimme's D3 correction for dispersion interactions ${ }^{36}$ was used to describe the exchange and correlation electronic interactions. The projected augmented wave method ${ }^{37,38}$ was used to build the pseudopotentials. Convergence criteria for the electronic selfconsistent loop and the conjugate gradient algorithm was set to $10^{-5} \mathrm{eV}$ and 0.0 $\mathrm{eV} \AA^{-1}$, respectively. Due to the large size of the BrparaImeta-TP and Ipar$a \mathbf{B r}$ meta-TP molecules we studied the halogen bonds of a system constituted by smaller bromobenzene and iodobenzene molecules deposited on a three-layer $\mathrm{Cu}$ (111) slab. Since interactions with the subsurface atoms are important for the adsorption conformation of the molecules, including several $\mathrm{Cu}(111)$ layers is crucial $^{40,45,48,49}$. These halobenzene molecules adsorbed on a triangular arrangement with the halogen atoms on bridge positions and the benzene rings on fcchollow positions are suitable models to accurately reproduce the experimental observations.

In addition, we use our recently developed method to simulate high resolution AFM images ${ }^{41-43}$. This method is able to produce theoretical AFM images at different heights by constructing a potential using some inputs from previous DFT calculations, essentially the total charge densities of the tip and the sample, and the electrostatic potential of the sample. The method just requires fitting two parameters to get a proper description of the Pauli repulsion given by

$V_{\mathrm{SR}}=V_{0} \int\left[\rho^{\text {tip }}\left(\boldsymbol{r}, R_{\text {tip }}\right) \rho^{\text {sam }}(\boldsymbol{r})\right]^{\alpha} d \boldsymbol{r}$. Here, they were set to

$V_{0}=32.7[\mathrm{eV}], \alpha=1.11$. The relaxation of the CO probe was accounted through a tilt potential, $V_{\text {tilt }}=\frac{1}{2} k^{\prime}\left(\Delta x^{2}+\Delta y^{2}\right)$, where $k^{\prime}$ was set to $0.46 \mathrm{~J} \mathrm{~m}^{-2}$. Notice that, following the approach introduced by Hapala et al..$^{50}$, we have minimized the probe position approaching the CO-sample interaction for those given by a rigid tip and restraining it to the original plane.

Please find more information about the DFT calculations and other computational details in the Supplementary information.

\section{Data availability}

The data supporting the findings of this study are available within the paper and its Supplementary Information files.

Received: 13 May 2020; Accepted: 1 October 2020;

Published online: 06 November 2020

\section{References}

1. Metrangolo, P. \& Resnati, G. Halogen bonding: a paradigm in supramolecular chemistry. Chemistry 7, 2511-2519 (2001).

2. Gilday, L. C. et al. Halogen bonding in supramolecular chemistry. Chem. Rev. 115, 7118-7195 (2015)

3. Zheng, Q.-N. et al. Formation of halogen bond-based 2D supramolecular assemblies by electric manipulation. J. Am. Chem. Soc. 137, 6128-6131 (2015).

4. Niyas, M. A., Ramakrishnan, R., Vijay, V., Sebastian, E. \& Hariharan, M. Anomalous halogen-halogen interaction assists radial chromophoric assembly. J. Am. Chem. Soc. 141, 4536-4540 (2019).

5. Mukherjee, A., Tothadi, S. \& Desiraju, G. R. Halogen bonds in crystal engineering: like hydrogen bonds yet different. Acc. Chem. Res. 47, 2514-2524 (2014).

6. Albright, E., Cann, J., Decken, A. \& Eisler, S. Halogen ‥halogen interactions in diiodo-xylenes. CrystEngComm 19, 1024-1027 (2017).

7. Mukherjee, A. et al. Halogenated building blocks for 2D crystal engineering on solid surfaces: lessons from hydrogen bonding. Chem. Sci. 10, 3881-3891 (2019).

8. Sutar, R. L. \& Huber, S. M. Catalysis of organic reactions through halogen bonding. ACS Catal. 9, 9622-9639 (2019).

9. Lu, Y. et al. Halogen bonding-a novel interaction for rational drug design? J. Med. Chem. 52, 2854-2862 (2009).

10. Kolar, M., Hobza, P. \& Bronowska, A. K. Plugging the explicit sigma-holes in molecular docking. Chem. Commun. 49, 981-983 (2013).

11. Clark, T., Hennemann, M., Murray, J. S. \& Politzer, P. Halogen bonding: the sigma-hole. J. Mol. Model. 13, 291-296 (2007)

12. Desiraju, G. R. \& Parthasarathy, R. The nature of halogen $\cdots$ halogen interactions: are short halogen contacts due to specific attractive forces or due to close packing of nonspherical atoms? J. Am. Chem. Soc. 111, 8725-8726 (1989).

13. Desiraju, G. R. et al. Definition of the halogen bond (IUPAC Recommendations 2013). Pure Appl. Chem. 85, 1711-1713 (2013).

14. Desiraju, G. R. \& Harlow, R. L. Cyano-halogen interactions and their role in the crystal-structures of the 4-halobenzonitriles. J. Am. Chem. Soc. 111, 6757-6764 (1989).

15. Cavallo, G. et al. The halogen bond. Chem. Rev. 116, 2478-2601 (2016).
16. Riley, K. E. et al. Halogen bond tunability I: the effects of aromatic fluorine substitution on the strengths of halogen-bonding interactions involving chlorine, bromine, and iodine. J. Mol. Model. 17, 3309-3318 (2011).

17. Gross, L., Mohn, F., Moll, N., Liljeroth, P. \& Meyer, G. The chemical structure of a molecule resolved by atomic force microscopy. Science 325, 1110-1114 (2009).

18. Chiang, C.-1, Xu, C., Han, Z. \& Ho, W. Real-space imaging of molecular structure and chemical bonding by single-molecule inelastic tunneling probe. Science 344, 885-888 (2014).

19. Kawai, S. et al. Extended halogen bonding between fully fluorinated aromatic molecules. ACS Nano 9, 2574-2583 (2015).

20. Han, Z. et al. Imaging the halogen bond in self-assembled halogenbenzenes on silver. Science 358, 206-206 (2017).

21. Gallardo, A., Fanfrlík, J., Hobza, P. \& Jelínek, P. Nature of binding in planar halogen-benzene assemblies and their possible visualization in scanning probe microscopy. J. Phys. Chem. C 123, 8379-8386 (2019).

22. Strohmaier, R., Ludwig, C., Petersen, J., Gompf, B. \& Eisenmenger, W. Stm investigations of C6Br6 on Hopg and MoS2. Surf. Sci. 318, L1181-L1185 (1994).

23. Walch, H., Gutzler, R., Sirtl, T., Eder, G. \& Lackinger, M. Material- and orientation-dependent reactivity for heterogeneously catalyzed carbonbromine bond homolysis. J. Phys. Chem. C 114, 12604-12609 (2010).

24. Wang, W., Shi, X., Wang, S., Hove, M. A. V. \& Lin, N. Single-molecule resolution of an organometallic intermediate in a surface-supported ullmann coupling reaction. J. Am. Chem. Soc. 133, 13264-13267 (2011).

25. Shang, J. et al. Assembling molecular Sierpinski triangle fractals. Nat. Chem. 7, 389-393 (2015).

26. Chung, K.-H. et al. Polymorphic porous supramolecular networks mediated by halogen bonds on $\operatorname{Ag}(111)$. Chem. Commun. 47, 11492-11494 (2011).

27. Yoon, J. K. et al. Visualizing halogen bonds in planar supramolecular systems J. Phys. Chem. C 115, 2297-2301 (2011).

28. Huang, $\mathrm{H}$. et al. Competition between hexagonal and tetragonal hexabromobenzene packing on $\mathrm{Au}(111)$. ACS Nano 10, 3198-3205 (2016)

29. Zhang, $H$. et al. Two-dimensional chirality transfer via on-surface reaction. $J$ Am. Chem. Soc. 138, 11743-11748 (2016).

30. $\mathrm{Li}, \mathrm{Q}$. et al. Self-assembly directed one-step synthesis of [4] radialene on $\mathrm{Cu}$ (100) surfaces. Nat. Commun. 9, 3113 (2018).

31. Zint, S. et al. Imaging successive intermediate states of the on-surface Ullmann reaction on $\mathrm{Cu}(111)$ : role of the metal coordination. ACS Nano 11, 4183-4190 (2017).

32. Ebeling, D. et al. Adsorption structure of mono- and diradicals on a $\mathrm{Cu}(111)$ surface: chemoselective dehalogenation of 4-bromo-3'-iodo-. ACS Nano 13, 324-336 (2019)

33. Fan, Q. et al. Nanoribbons with nonalternant topology from fusion of polyazulene: carbon allotropes beyond graphene. J. Am. Chem. Soc. 141, 17713-17720 (2019).

34. Kresse, G. \& Furthmüller, J. Efficient iterative schemes for ab initio totalenergy calculations using a plane-wave basis set. Phys. Rev. B 54, 11169 (1996).

35. Perdew, J. P., Burke, K. \& Ernzerhof, M. Generalized gradient approximation made simple. Phys. Rev. Lett. 77, 3865-3868 (1996).

36. Grimme, S., Antony, J., Ehrlich, S. \& Krieg, H. A consistent and accurate ab initio parametrization of density functional dispersion correction (DFT-D) for the 94 elements H-Pu. J. Chem. Phys. 132, 154104-154104 (2010).

37. Blöchl, P. E. Projector augmented-wave method. Phys. Rev. B 50, 17953-17979 (1994)

38. Kresse, G. \& Joubert, D. From ultrasoft pseudopotentials to the projector augmented-wave method. Phys. Rev. B 59, 1758-1775 (1999).

39. Björk, J., Hanke, F. \& Stafström, S. Mechanisms of halogen-based covalent self-assembly on metal surfaces. J. Am. Chem. Soc. 135, 5768-5775 (2013).

40. Zhong, Q. et al. Symmetry breakdown of $4,4^{\prime}$-diamino-p-terphenyl on a $\mathrm{Cu}$ (111) surface by lattice mismatch. Nat. Commun. 9, 3277-3277 (2018)

41. Ellner, M. et al. The electric field of co tips and its relevance for atomic force microscopy. Nano Lett 16, 1974-1980 (2016).

42. Ellner, M., Pou, P. \& Pérez, R. Atomic force microscopy contrast with CO functionalized tips in hydrogen-bonded molecular layers: Does the real tip charge distribution play a role? Phys. Rev. B 96, 075418-075418 (2017).

43. Ellner, M., Pou, P. \& Pérez, R. Molecular identification, bond order discrimination, and apparent intermolecular features in atomic force microscopy studied with a charge density based method. ACS Nano 13 , 786-795 (2019)

44. Ebeling, D. et al. Chemical bond imaging using higher eigenmodes of tuning fork sensors in atomic force microscopy. Appl. Phys. Lett. 110, 183102 (2017).

45. Zint, S., Ebeling, D., Ahles, S., Wegner, H. A. \& Schirmeisen, A. Subsurfacecontrolled angular rotation: triphenylene molecules on $\mathrm{Au}(111)$ substrates. J. Phys. Chem. C 120, 1615-1622 (2016)

46. Giessibl, F. J. High-speed force sensor for force microscopy and profilometry utilizing a quartz tuning fork. Appl. Phys. Lett. 73, 3956-3958 (1998). 
47. Bartels, L., Meyer, G. \& Rieder, K. H. Controlled vertical manipulation of single $\mathrm{CO}$ molecules with the scanning tunneling microscope: a route to chemical contrast. Appl. Phys. Lett. 71, 213-215 (1997).

48. Ohmann, R., Levita, G., Vitali, L., De Vita, A. \& Kern, K. Influence of subsurface layers on the adsorption of large organic molecules on closepacked metal surfaces. ACS Nano 5, 1360-1365 (2011).

49. Mollenhauer, D., Gaston, N., Voloshina, E. \& Paulus, B. Interaction of pyridine derivatives with a gold (111) surface as a model for adsorption to large nanoparticles. J. Phys. Chem. C 117, 4470-4479 (2013).

50. Hapala, P. et al. Mechanism of high-resolution STM/AFM imaging with functionalized tips. Phys. Rev. B 90, 085421-085421 (2014).

\section{Acknowledgements}

Financial support by the Deutsche Forschungsgemeinschaft via the grants EB535/1-1, EB535/2-1, SCHI 619/13, and the GRK (Research Training Group) 2204 "Substitute Materials for Sustainable Energy Technologies" is gratefully acknowledged. We thank the financial support from the Spanish MINECO (AEI/FEDER, UE, project MAT201783273-R), from MICINN through the "María de Maeztu" Program for Units of Excellence in R\&D (CEX2018-000805-M), from Comunidad de Madrid Industrial Doctorate 2017 (reference IND2017/IND-7793), and from Quasar Science Resources S.L. Computer time provided by the Red Española de Supercomputación (RES) at the Marenostrum Supercomputer is also acknowledged. P.H., T.S., and D.M. acknowledge the support by the administrators of the YACANA Cluster and the IT Centre (HRZ) of the Justus-Liebig University Giessen.

\section{Author contributions}

D.E. conceived the project. J.T., Q.Z., and D.M.J. carried out the experiments. J.C.C., C.R.M., P.H., T.S., D.M., P.P., and R.P. performed the DFT computations. S.A. and H.A.W. are responsible for the synthesis of the molecules. D.M., H.A.W., P.P., R.P., and A.S. contributed significantly to the scientific discussion of the results. All authors commented on the paper that was drafted by D.E.

\section{Funding}

Open Access funding enabled and organized by Projekt DEAL.

\section{Competing interests}

The authors declare no competing interests.

\section{Additional information}

Supplementary information is available for this paper at https://doi.org/10.1038/s41467 020-19379-4.

Correspondence and requests for materials should be addressed to D.E.

Peer review information Nature Communications thanks the anonymous reviewer(s) for their contribution to the peer review of this work.

Reprints and permission information is available at http://www.nature.com/reprints

Publisher's note Springer Nature remains neutral with regard to jurisdictional claims in published maps and institutional affiliations.

(c) ()

Open Access This article is licensed under a Creative Commons Attribution 4.0 International License, which permits use, sharing, adaptation, distribution and reproduction in any medium or format, as long as you give appropriate credit to the original author(s) and the source, provide a link to the Creative Commons license, and indicate if changes were made. The images or other third party material in this article are included in the article's Creative Commons license, unless indicated otherwise in a credit line to the material. If material is not included in the article's Creative Commons license and your intended use is not permitted by statutory regulation or exceeds the permitted use, you will need to obtain permission directly from the copyright holder. To view a copy of this license, visit http://creativecommons.org/ licenses/by/4.0/.

(C) The Author(s) 2020 